\title{
Incendios, sucesión y restauración ecológica en contexto
}

\author{
Wildland fires, sucession and \\ ecological restoration in context
}

Fabian M. Jaksic ${ }^{1}$ \& José M. Fariña ${ }^{1}$

\section{Resumen}

En el presente trabajo entregamos un marco conceptual sobre el efecto del fuego en la estructura y dinámica de ecosistemas de bosques, matorrales y praderas, el cual puede ser utilizado para evaluar opciones de restauración ecológica de dichos ecosistemas. Para ello, revisamos la información histórica sobre los incendios en distintos ecosistemas chilenos, con especial énfasis en los casos de la Patagonia y Torres del Paine. Luego revisamos la evidencia relacionada con la sucesión que ocurre luego de los incendios, los cambios ecosistémicos y las principales características biológicas de la vegetación que están asociadas a esta dinámica ecológica. Finalmente, en base a una revisión de los principales conceptos asociados a la restauración ecológica y su contexto interdisciplinario, concluimos con una serie de recomendaciones que podrian servir de guía para eventuales planes de restauración, e incluso de manejo y conservación de los ecosistemas de la Patagonia y Torres del Paine que han sido afectados por el fuego.

\section{Palabras clave:}

Patagonia, Torres del Paine, sucesión ecológica, Fuego.

\footnotetext{
Abstract

In this article we construct a theoretical framework about the effect of fire on the structure and dynamics of forests, shrublands and grasslands, which can be used to address the ecological restoration of these ecosystems. To do this, we review the historical information of fires that have
}

affected diverse Chilean ecosystems with emphasis on the cases of Patagonia and Torres del Paine. Afterward, we analyze the main aspects of the ecological succession occurring after the fire, the main ecosystem changes, and the biological characteristics of vegetation in relation with fire perturbations. Finally, on reviewing the main aspects of ecological restoration and its interdisciplinary context, we issue recommendations for eventual plans of restoration, management, and conservation for Patagonia in general and for Torres del Paine in particular.

\section{Key words:}

Patagonia, Torres del Paine, ecological succession, Fire.

\section{INTRODUCCIÓN}

Este trabajo se inspira en el creciente papel que los incendios de bosques, matorrales y pastizales tienen sobre estas formaciones vegetales y los ecosistemas de los cuales forman parte. Al contrario de la mayoría del trabajo científico, orientado a la puesta a prueba de hipótesis que parten desde un contexto general a una aplicación a circunstancias específicas, aquí procedemos al revés. Usando como ejemplo la situación de la vegetación chilena en relación a los incendios,

1 Centro de Ecología Aplicada \& Sustentabilidad (CAPES), Pontificia Universidad Católica de Chile, Santiago, Chile.fjaksic@bio.puc.cl $\longrightarrow$ 
construimos un marco conceptual que busca encontrar lecciones generales asociadas con la perturbación por fuego y la sucesión ecológica, a diversas escalas de resolución. Nuestro objetivo es proveer un contexto dentro del cual evaluar opciones de restauración ecosistémica, específicamente para el caso de Patagonia en general y del Parque Nacional Torres del Paine en particular.

\section{INCENDIOS: LOS CASOS DE CHILE, PATAGONIA Y TORRES DEL PAINE}

Chile: Hay controversia sobre los regímenes históricos de fuegos naturales en diferentes ecosistemas chilenos. Se ha reportado que sólo el $0,1 \%$ de los incendios registrados en los últimos 20 años, desde Chile central a la Patagonia, podrían deberse a causas naturales (Armesto et al. 2009; Fernández et al. 2010). Aún cuando se ha postulado que algunos incendios ocurrieron naturalmente en algunas comunidades mediterráneas y templadas de Chile, desde antes de la llegada del ser humano (Fuentes \& Espinoza, 1986; Abarzúa \& Moreno, 2008), la frecuencia de incendios no parece haber sido suficientemente alta como para promover adaptaciones de resistencia o tolerancia en la mayoría de las especies de plantas. En este contexto, prácticamente no existen, salvo raras excepciones, ecosistemas dependientes del fuego en Chile (Montenegro et al. 2004). La vegetación nativa chilena en la zona centro-sur no ha desarrollado adaptaciones especializadas para resistir incendios o regenerar post-incendios (Armesto et al. 1995), salvo algunas especies que por sus ciclos de vida longevos poseen mecanismos de resistencia a incendios de baja frecuencia asociados a fenómenos volcánicos (Donoso 1993; Veblen et al. 1995; Gonzáles \& Veblen, 2006).

Patagonia: Los incendios forestales en los ecosistemas de la Patagonia, tanto chilena como argentina, están fuertemente influenciados por la variabilidad climática interanual. Se ha observado que años con mayor actividad de incendios están usualmente asociados a inviernos secos y veranos cálidos (Veblen et al. 1999). Aunque existe evidencia de que esta zona es afectada por incendios desde hace milenios, los estudios sugieren que en su mayoría estos eventos estuvieron relacionados con prácticas de los indígenas que poblaron el lugar y el posterior arribo de los colonizadores europeos que abrieron tierras para la ganadería, siendo escasos los incendios provocados por causas naturales (Heusser, 1987, 1994).

Con la colonización europea de la Patagonia, durante la segunda mitad del siglo XIX, los incendios se volvieron más recurrentes (Veblen et al. 1999), debido al uso del fuego que hicieron los colonos (Lara et al. 2003; Armesto et al. 2009). La impenetrable estructura de muchos bosques, con sus compleja estratificación vertical, y sus largos períodos de rotación hicieron que el bosque nativo fuera visto como un escollo para el desarrollo de la agricultura y la ganadería, y por este motivo grandes extensiones de terreno fueron incendiadas, algunas durante meses e incluso años (Sanhueza, 2001). La influencia humana presente desde el período pre-hispánico al europeo, sumado a la alta presión de herbivoría por ganado, que generó un aumento en la frecuencia de eventos de incendios, tiene el potencial de transformar los paisajes de manera radical, remplazando bosques deciduos centenarios por pampas, estepas y matorrales de manera irreversible en la medida que las perturbaciones se mantienen (Tortorelli, 1947). Los matorrales generados podrían poseer especies arbustivas y herbáceas que rebrotarian vigorosamente después de los incendios, posiblemente sujetas a fuerte selección por el impacto recurrente del fuego desde la ocupación humana de la región. Sin embargo, los ecosistemas de bosques deciduos, dominados principalmente por especies dependientes de regeneración a partir de semillas (e.g., lenga, coihue de Magallanes), tienen regeneración escasa o nula tras incendios severos, siendo reemplazados por comunidades de estepa. Durante el siglo XX, la sustitución del bosque, especialmente de lenga, se vio acelerada en toda la Patagonia producto de los incendios intencionales para la producción de pasturas (Mermoz et al. 2005; Veblen et al. 2011).

Son varios los mecanismos asociados a la ocurrencia de fuego en la historia de la Patagonia. Se ha descrito actividad volcánica, clima, cambios vegetacionales y perturbación humana (tanto nativa como europea), durante los últimos 11.500 años (Heusser, 1994; Markgraf \& Anderson, 1994; Huber et al. 2004; Moreno, 2004; Whitlock et al. 
2006; Abarzua \& Moreno, 2008). Los patrones espaciales y temporales de fuego son altamente susceptibles a variabilidad climática e impacto humano, los cuales afectan tanto la frecuencia de ignición como las características del combustible. La propagación de los fuegos requiere de la combinación de un bajo contenido de humedad del combustible, y una elevada acumulación y continuidad del combustible, además de una condición climática seca.

La ocurrencia del fuego se ve limitada por factores primarios que pueden variar fuertemente a lo largo de gradientes ambientales (e.g., Kitzberger et al. 1997; Veblen et al. 1999). En Patagonia norte los eventos de fuego difieren a lo largo del gradiente de humedad efectiva oeste-este a través de los Andes. Los combustibles leñosos del bosque lluvioso requieren de periodos de sequías para llegar a quemarse y son controlados por la humedad del combustible. Por otra parte, los combustibles finos generados en ambientes de estepa son rápidamente desecados y son lo bastante secos como para soportar fuegos aún durante años húmedos, pero de menor escala. Durante el siglo XX, la frecuencia de fuego y las fuentes de ignición estuvieron mucho más vinculadas a la perturbación humana, en conjunción con las variaciones climáticas. Registros sedimentarios de partículas de carbón fósil muestran que el fuego ha sido un importante factor de perturbación de los ecosistemas templados de Patagonia a través de diferentes intervalos de tiempo durante los últimos 15.000 años (Markgraf \& Anderson, 1994; Heusser 1995; Huber et al. 2004; Villa-Martínez \& Moreno, 2007). Esto significa que independiente de la influencia actual del ser humano, estos registros muestran que el fuego ha sido un importante factor de perturbación en Patagonia, desde el término de la última glaciación.

También se ha reportado que períodos de alta variabilidad climática en Patagonia, son factores incidentes en el aumento de la actividad del fuego a escala de tiempo multi-milenial (Markgraf \& Anderson, 1994). Por otra parte, se sugiere como causa primaria del fuego a los cazadores paleo-indios como agentes de ignición; por ello se propuso el uso de estratigrafías de carbón como indicadores de presencia humana y como un trazador de rutas de migración de cazadores-recolectores prehistóricos (Heusser, 1994). Aun así, la falta de datos arqueológicos no permite determinar con certeza si todos los casos de aumento de actividad de fuego se asocian a ocupaciones humanas. Actualmente, se apoya la sugerencia de que el clima ha sido el principal agente causal del fuego en la región patagónica a escala milenial (Huber et al. 2004; Whitlock et al. 2007; Power et al. 2008).

Torres del Paine: El Parque Nacional Torres del Paine (XII región, Chile) dispone de registros de partículas de carbón, que muestran similitud con los estudios regionales de la historia del fuego descritos más arriba. Los trabajos científicos reportados en base a análisis de partículas microscópicas de pantanos localizadas en partes bajas del Parque muestran la siguiente secuencia de eventos por sitio:

Sitio Torres del Paine (al este del margen oriental de Lago Sarmiento) evidencia elevada actividad de fuego entre 12.800 y 9.000 años AP (antes del presente), baja actividad entre 9.000 y 6.000 años AP, un incremento sostenido en la actividad de fuego a partir de los 6.000 años $\mathrm{AP}$, seguido por fluctuaciones de gran magnitud durante los últimos 3.000 años (Heusser, 1995).

Sitio Vega Ñandú (9 km al noroeste del sitio de Heusser, 1995) muestra baja actividad de fuego durante todo el registro, pero es notable el incremento moderado de la actividad entre 10.8007.000 años AP, y al mismo tiempo un incremento en las proporciones de elementos arbóreos. Esta fase fue seguida por máximos de fuego de corta duración centrados entre 5.700 y 5.200 años AP, y un máximo entre 2.300 y 2.000 años AP (VillaMartínez \& Moreno, 2007).

Sitio Lago Guanaco evidencia elevada actividad de fuego entre 13.000 y 10.000 años $\mathrm{AP}$, seguido por un descenso de valores que está puntuado por máximos de carbón entre 3.3002.800, 1.900-1.200 años AP. Las diferencias entre los registros que provienen del Parque pueden reflejar la existencia de diferencias en los regímenes de fuego cercanos a cada sitio, o diferencias sitioespecíficas en polen fósil, estratigrafía y control 
cronológico (Moreno et al. 2009).

En resumen, todos los registros que provienen del Parque muestran un incremento pronunciado de la actividad de fuego durante los últimos 100-200 años. Estos sugieren nuevamente que el clima tiene un rol importante sobre los patrones regionales de la historia del fuego en Patagonia. Los trabajos de Villa-Martínez \& Moreno (2007), sobre las reconstrucciones de la historia de la vegetación en el Parque, sugieren que este último pulso de incremento de fuego (100200 AP) está asociado a una abrupta reducción del indicador de bosque (polen de Nothofagus) y un incremento del indicador de perturbación humana Rumex y Poáceas (pastos). La aparición de Rumex sugiere que la reducción del bosque y el incremento del fuego fueron la causa y efecto de una rápida (25-50 años) y marcada transformación del paisaje debido al establecimiento de colonos humanos en esta área, lo cual permitió el desarrollo del actual paisaje dominado por estepas y pampas. Así, la magnitud y rapidez de la pérdida de cobertura de bosques ilustra su vulnerabilidad a una actividad de fuego recurrente.

\section{SUCESIÓN POST-INCENDIO}

Entre las perturbaciones más frecuentes e importantes que afectan los ecosistemas templados del planeta, encontramos el fuego (e.g., Clark \& Richard, 1996; Kitzberger et al. 1997; Long et al. 1998), tanto en presencia como en ausencia de sociedades humanas aledañas (Bond \& Wilgen, 1996).

El fuego puede ser originado naturalmente o por efecto del hombre. La variabilidad e importancia que los regímenes de incendios naturales tienen en la dinámica de numerosos ecosistemas, ha sido bien reportada (Goldamer, 1991; Shlisky et al. 2007). También se sabe que las actividades humanas han modificado la incidencia, intensidad y extensión de los fuegos en todas las regiones del mundo. En algunos casos la gestión de prevención y combate de incendios, especialmente en zonas pobladas, ha llevado a un cese de este agente perturbador natural, causando efectos negativos sobre la dinámica de los ecosistemas y su biodiversidad. Por otra parte, en muchos ecosistemas donde la presencia del fuego antes del arribo de los seres humanos era escasa o inexistente, el impacto humano y el desarrollo de las grandes urbes y la sociedad industrial ha provocado un considerable aumento de la frecuencia de incendios. Aún en lugares remotos, o protegidos del impacto humano, tales como parques naturales, la concurrencia de visitantes y exploradores puede causar grandes catástrofes debido al difícil control de los incendios en localidades inaccesibles.

Las repercusiones que puede tener el fuego sobre los ecosistemas son numerosas y dependen de la historia de eventos de fuego, tanto antes como después de la ocupación humana. El fuego puede tener un rol positivo o negativo en los ecosistemas. Ecosistemas tales como sabanas y bosques de coníferas boreales dependen del fuego en los procesos físicos y biológicos de renovación de suelo y regeneración de la vegetación, diversificando el paisaje con un efecto positivo sobre los ciclos biogeoquímicos y productividad involucrados (Flannigan et al. 2001). Por otra parte, hay ecosistemas donde los incendios históricos no han sido frecuentes, y pueden alterar profundamente la comunidad de plantas existente y los ecosistemas, modificando la proporción de formas de vida de las plantas, reduciendo la biodiversidad a aquellas especies más resistentes al impacto del fuego, creando condiciones para el reemplazo de especies nativas por exóticas, y alterando los patrones sucesionales de recuperación de la cobertura vegetal (De Bano et al. 1998).

Asimismo, las distintas especies vegetales presentes en los distintos ecosistemas responden de forma diferencial al fuego, dependiendo de sus habilidades para tolerarlo y de los mecanismos de regeneración que posean (Lloret \& Vilà, 2003). En especies carentes de adaptaciones de resistencia o tolerancia al fuego, los incendios, incluso cuando su intensidad es baja, pueden causar una alta mortalidad. Si estas adaptaciones están ausentes en gran parte de las especies que componen la comunidad, el impacto sobre el ecosistema puede cambiar drásticamente la dinámica y composición de la vegetación y el resto de la cadena trófica. Así, el mantenimiento de las poblaciones, y la comunidad en general, depende exclusivamente de la resistencia de las semillas en el suelo, o presentes en las plantas, de la capacidad de rebrote de las 
plantas dañadas, y de la recolonización del sitio desde sectores no afectados (Anderson, 1981; Whelan, 1995). Estos procesos de recolonización pueden tomar tiempos muy largos, en la medida que la capacidad de dispersión de la vegetación a partir de áreas no quemadas es muy limitada y los suelos han sido erosionados.

Por otra parte, los ecosistemas con historias recurrentes de incendios naturales, presentan generalmente especies que son capaces de aprovechar estos eventos de perturbación para regenerar y expandir sus poblaciones. En estos ecosistemas el fuego ha operado evolutivamente como un mecanismo de selección sobre especies de plantas y ha promovido el desarrollo de múltiples formas de regeneración post-fuego (Ford et al. 2000), e incluso en algunos casos la selección natural habría favorecido a aquellas especies más propensas a quemarse, creando comunidades dependientes de incendios (Mutch, 1970; Chang, 1996; Bond \& Keeley, 2005).

\section{RESTAURACIÓN ECOLÓGICA: DISTINCIONES NECESARIAS}

Entendemos por restauración ecológica aquellas acciones motivadas para recuperar un ecosistema alterado o modificado por agentes naturales o bien por obra del hombre. Este proceso busca reiniciar o apresurar el ecosistema perturbado ya sea en sus procesos, en su estructura comunitaria, o en su capacidad de resiliencia.

La alteración (degradación es un término con alta carga valórica) de un ecosistema se caracteriza por pérdidas en sus características originales, tales como su función o estructura, lo que puede dar lugar a un nuevo ecosistema, o restringir la persistencia del ecosistema original en el tiempo, por lo menos a escala de tiempo humana. Los factores que pueden causar la alteración de un ecosistema pueden ser eventos discretos, de un solo pulso, o recurrentes, de varios pulsos. A su vez, estos pueden ser de causados por un sólo factor o ser multifactoriales.

Cuando las alteraciones no ocurren de manera natural, la restauración ecológica es necesaria para "encausar" el ecosistema dañado o destruido a un estado lo más parecido posible al que existía antes de la alteración. Para esto es necesario conocer las condiciones previas a la alteración del ecosistema, tales como composición, estructura y función, ya que esto guiará el diseño de la restauración. Sin el estado basal de referencia de un ecosistema, la restauración ecológica es más difícil de implementar y se requieren herramientas de reconstrucción de la historia del ecosistema afectado (White \& Walker, 1997).

Si bien la restauración ecológica se basa, generalmente, en una comprensión del pasado, el objetivo no es reproducir un estado histórico determinado. La restauración no siempre recupera la composición y estructura del ecosistema previo, ya sea por limitaciones del conocimiento, o porque las condiciones actuales han cambiado y pueden definir una trayectoria de recuperación diferente, dando lugar a un ecosistema alternativo (cambio de fase). Asimismo, un objetivo realista de restauración ecológica es fomentar las condiciones que permiten la existencia de un ecosistema propio de la región en que se encuentra el área alterada, a través de proveer hábitats y características funcionales necesarias para mantener la dinámica del ecosistema original.

La restauración ecológica se lleva a cabo a través de acciones concretas tales como: (a) detener o menguar la ocurrencia de los eventos que originaron la alteración, (b) recuperar la flora y fauna nativa de los ecosistemas, (c) promover la sucesión ecológica o dinámica local estimulando la regeneración natural, (d) reponer procesos y funciones ecosistémicas, y (e) fomentar acciones de auto-recuperación que permitan al ecosistema sostener su recuperación en el tiempo. Estas acciones tienen por objetivo la resiliencia de un ecosistema, para que este pueda integrarse dentro de un paisaje más amplio dentro del contexto ecológico y cultural de la región.

Numerosos ecólogos que trabajan en restauración ecológica promueven facilitar el proceso de sucesión, a través de mecanismos similares a los procesos naturales, para formar comunidades en estados relativamente estables (McIntosh, 1980; Bradshaw, 1983; Dobson et al. 1997; Palmer et al. 1997; Whisenant, 1999; Young et al. 2001; Walker \& del Moral, 2003). Todos ellos han creado términos que denotan diferencias en énfasis y metodología. A continuación los más usados: 
Rehabilitación/Restauración: Ambos términos se refieren los mismos fines en el contexto de restauración ecológica, pero el énfasis de cada uno es diferente. En el caso de la rehabilitación, el objetivo es la restitución de la función ecológica y no la estructura, composición o diversidad original del ecosistema. Por lo cual en este caso es posible restablecer la función ecológica de la vegetación utilizando especies exóticas al lugar, las que no tienen relación con el ecosistema original. Para restauración ambiental, el objetivo central es el restablecimiento de una condición ambiental deseable para la sociedad, por ejemplo vegetación en una ladera, o la claridad y limpieza del agua de una laguna, más que la reposición de toda la complejidad y biodiversidad del ecosistema original.

Remediación/Reparación: Ambos términos refieren a actividades específicas, las que podrían ser parte de un programa de restauración ecológica, pero cuyos objetivos son más restringidos. El término remediación corresponde a la utilización de un proceso tecnológico o de ingeniería ambiental para reparar un tipo de daño específico; por ejemplo la limpieza de un área que haya sufrido un derrame de petróleo. Por otro lado, reparación se refiere a la sustitución de un ecosistema degradado por otro diferente al original mediante un trabajo de paisajismo o de ingeniería ambiental, con el fin de producir un ambiente con una condición estética o productiva deseada, y no necesariamente un ecosistema natural.

Plantación/Reforestación: Estos términos no son sinónimos de restauración ecológica, pero se usan para describir actividades específicas que podrían ser parte de un programa de restauración ecológica. Las plantaciones y la reforestación son formas de reponer la cobertura vegetal en un área donde la vegetación original ha sido removida, considerando sólo determinadas especies y no el ecosistema con toda su diversidad y complejidad.

Restauración pasiva/activa: La restauración pasiva es el conjunto de acciones que eliminan o modifican los factores que limitan la recuperación natural de un ecosistema, tales como corta, tala, caza, incendios intencionales, ganadería y pastoreo, de manera de facilitar la recuperación espontánea de los componentes biológicos y procesos ecológicos, a través del proceso de sucesión. Por otro lado, la restauración activa involucra acciones especificas que estimulen el desarrollo de la sucesión para lograr la restauración del ecosistema (Van Andel \& Aronson, 2006).

La forma más efectiva y eficiente en términos ecológicos y económicos de restaurar ecosistemas es a través de la restauración pasiva, con poca intervención. Sin embargo, debido a los recientes avances de la teoría de sucesión ecológica y su uso corriente en las prácticas de restauración, a veces por desconocimiento del proceso, la sucesión espontánea de la vegetación no es considerada una herramienta en muchos proyectos de restauración, donde dominan planteamientos tecnológicos. Para no gastar tiempo y recursos en acciones costosas y que conllevan resultados dudosos, es esencial hacer evaluaciones experimentales de la capacidad de los ecosistemas de recuperarse sin asistencia y generar modelos para predecir las trayectorias sucesionales posibles en los sitios restaurados (Morrison \& Lindell, 2010). Estas evaluaciones permiten orientar la selección de sitios prioritarios para la restauración en los cuales se puede obtener el óptimo balance entre el costo y el beneficio de las actividades a realizar, maximizando la probabilidad de éxito en el mediano y largo plazo.

\section{CONTEXTO INTERDISCIPLINARIO}

La restauración ecológica no sólo está vinculada con principios y experiencias de la ecología, sino también de las ciencias sociales, económicas, filosóficas, políticas y jurídicas, los cuales le confieren una dimensión amplia a la disciplina (Armesto et al. 2007). Estos aspectos son tan importantes como los antecedentes científicos y técnicos sobre el ecosistema perturbado y deben ser integrados en los proyectos de restauración (Comin et al. 2005).

Las ciencias sociales y económicas generalmente se vinculan a los proyectos de restauración ecológica ayudando a definir las metas, desde el punto de vista de las condiciones deseadas por la sociedad y los costos y beneficios económicos, sociales y culturales que implica esta tarea (Leopold et al. 2002). En este contexto, es 
crucial integrar las aspiraciones y conocimientos de las comunidades locales, contribuyendo a reconciliar intereses diversos y compartiendo entre todos los involucrados los costos y beneficios de los proyectos de restauración ecológica.

Con una visión holística, el diseño e implementación de proyectos de restauración incrementará la biodiversidad y la provisión de servicios ecosistémicos en ambientes alterados, especialmente donde los impactos han sido crónicos (Higgs, 2005). Este aumento de los servicios ecosistémicos, de producción, de regulación y culturales, generará beneficios directos e indirectos para las comunidades cercanas y para la sociedad en su conjunto. Algunos de los beneficios sociales y económicos que genera la restauración son el aumento de ingresos y la creación de empleos a través de la producción de bienes, servicios ambientales y turismo en ecosistemas que se encontraban alterados. Asimismo, las áreas restauradas pueden transformarse en espacios de recreación, educación y otros servicios para las comunidades locales.

Por otra parte, las disciplinas de la ética y la filosofía contribuyen a una mayor conciencia pública y privada de las consecuencias de la alteración/ degradación ambiental y la forma de combatirla o contenerla (Cowell, 1993). Ellas pueden ayudar a decidir el establecimiento de marcos legales o sociales, que obliguen o fomenten que personas, empresas o agencias estatales que causen alteración o daño a los ecosistemas, o quienes se benefician de los ecosistemas en su condición natural, incluyan y financien proyectos de restauración ecológica y reparación/compensación ambiental (Aronson et al. 2006).

\section{CONCLUSIONES}

Tomando en cuenta los antecedentes sobre incendios forestales tanto en la Patagonia como en Torres del Paine y el marco conceptual de la ecología de la restauración expuesto anteriormente, es posible sugerir dos escenarios para la dinámica de cambios ecosistémicos que han sufrido ambos paisajes así como las perturbaciones que han sido asociadas a dichos cambios, $e$ incluso perturbaciones que aún no han sido evidenciadas

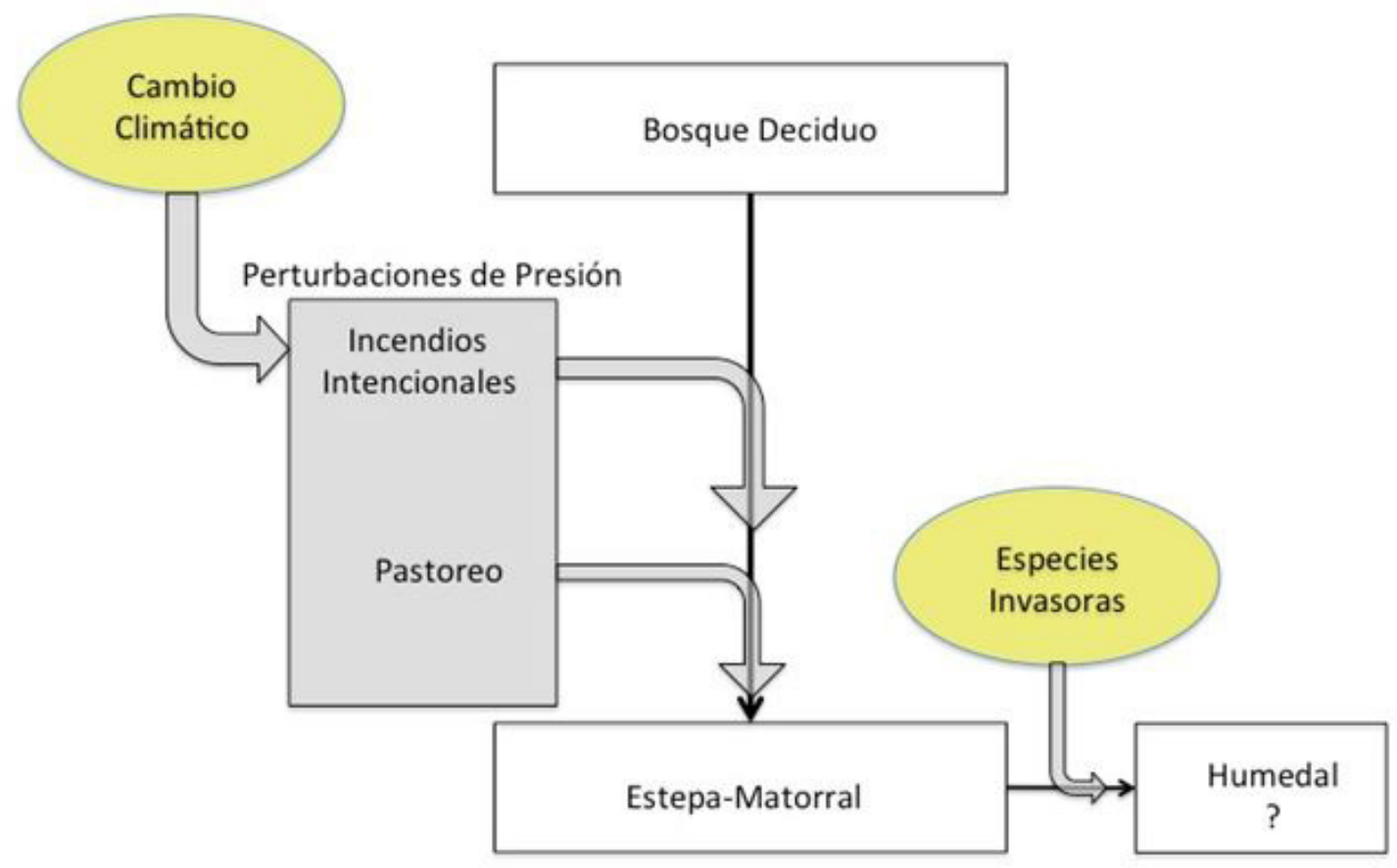

Fig. 1. Situación actual y dinámica de cambios ecosistémicos en la Patagonia y las perturbaciones de presión asociadas a dichos cambios. Se indican además las posibles trayectorias en respuesta al cambio climático y la introducción de especies invasoras tales como el castor. 


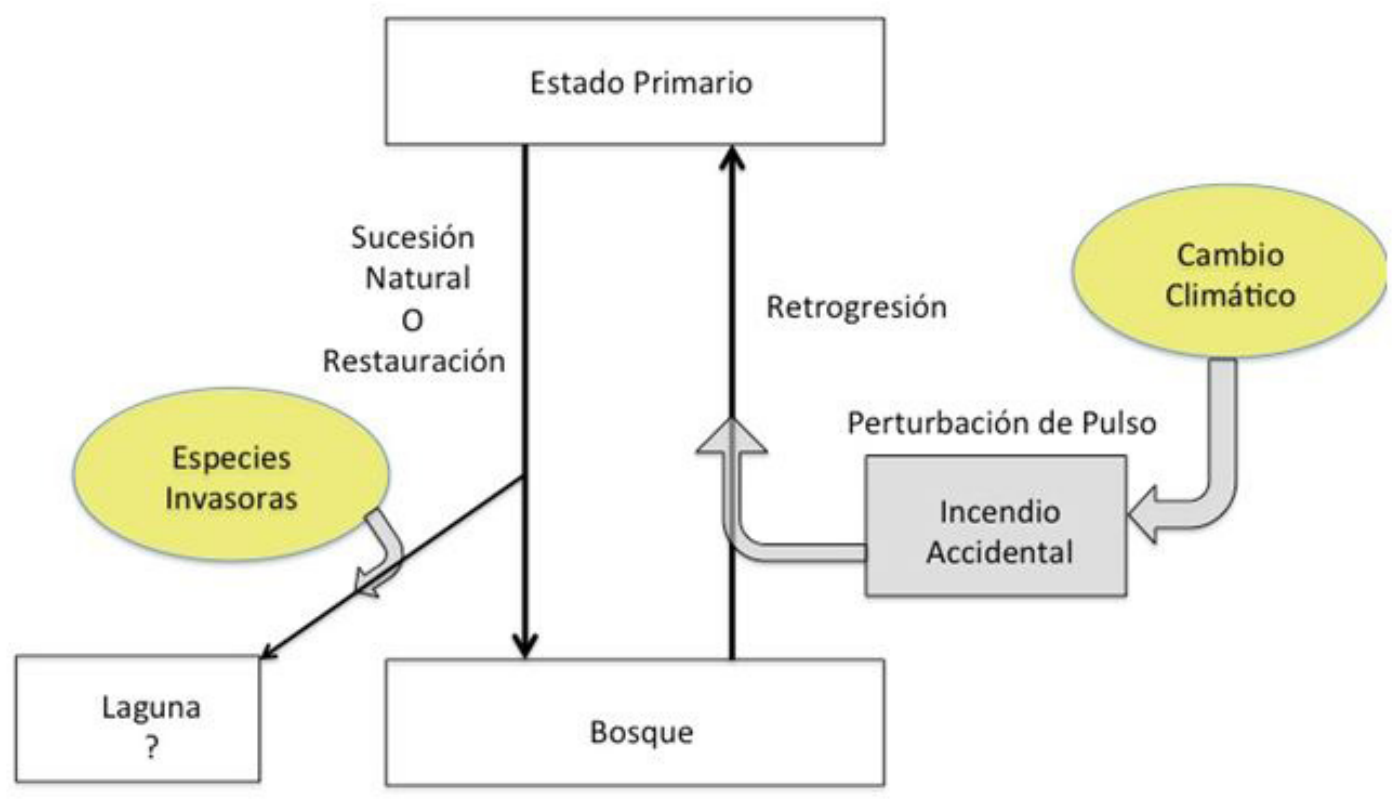

Fig. 2. Situación actual y dinámica de cambios ecosistémicos en Torres del Paine y la perturbación de pulso asociada a dichos cambios. Se indican además las posibles trayectorias en respuesta al cambio climático y la introducción de especies invasoras tales como el castor.

y que podrían jugar un rol preponderante en las dinámicas ecosistémicas en cuestión.

Patagonia: Para el caso de la Patagonia, reconocemos que el paisaje ha sido históricamente $e$ irreversiblemente modificado a través del uso frecuente del fuego (incendios intencionales) derivando en el estado de Estepa y Matorrales, los cuales son mantenidos a través, y para, el pastoreo (presión de herbivoría) (Fig. 1). En este caso no reconocemos la necesidad de alguna medida de restauración ni pasiva ni activa, pero si nos resulta preocupante el posible efecto que tendría a futuro sobre esta dinámica el cambio climático global, que para la región incrementará la incidencia de inviernos secos y veranos cálidos. Muy probablemente, de cumplirse las predicciones de los modelos de cambio climático, se acentuará la frecuencia de incendios en esta zona "atrapando" al sistema en su condición de Estepa o Matorral. Por otra parte, para esta misma zona, un componente que puede generar cierta incertidumbre, por el hecho de producir una situación distinta a la de Estepa, es el ingreso de especies invasoras bio-ingenieras tales como el castor (Anderson et al. 2006, 2009; Wallem et al. 2007; Henn et al. 2014). En este caso, el ingreso del castor, cambiaria los cursos de agua incrementando las áreas inundadas con lo cual, es probable que el sistema de Estepa-Matorral incorpore a especies de flora y fauna de hábitos acuáticos, tales como plantas palustres y aves, derivando en un tercer sistema de mosaico entre zonas inundadas (humedales) y Estepa-Matorral. Esta predicción está en línea con lo documentado por Anderson et al. (2009) para ecosistemas riparianos-forestales de Tierra del Fuego.

Torres del Paine: En este caso (Fig. 2), reconocemos que amplias extensiones de bosque fueron afectadas accidentalmente (perturbación de pulso) por el fuego recientemente, sin que dicha perturbación haya provocado un cambio de estado ecosistémico sino que más bien un retorno hacia los estadios sucesionales tempranos (i.e., retrogresión) de dichos bosques, con lo cual es escenario resultante es reversible a través de medidas de restauración. La restauración en este caso sería pasiva, poniendo especial énfasis en la eliminación de los factores que limitan la recuperación (i.e., mayor control sobre las amenazas de nuevos incendios accidentales), pero también, dado que el bosque original no está compuesto de 
especies que presenten adaptaciones o resistan al fuego (se eliminaron los propágulos) podría ser acompañada de medidas de reforestación de las especies dominantes que conformaban los bosques originales. Gran parte de este trabajo debiese ser apoyado por referencias de la sucesión que ocurrió en los bosques deciduos de la región luego de la ocurrencia de incendios (Abarzúa \& Moreno, 2008). En este caso, al igual que el anterior, es necesario tomar en cuenta las predicciones del cambio climático para la región, así como también el efecto que tendría el ingreso de especies invasoras como el castor. De ingresar el castor, la alteración de los cursos de agua podría provocar la inundación de los bosques en recuperación con lo cual se irán eliminando paulatinamente las áreas en recuperación, sacándolas de la vía de sucesión esperada desde el estado retrogresivo en que se encuentran hacia un estado de bosque inundado $y$ posteriormente humedal o laguna.

\section{AGRADECIMIENTOS} 2014.

Este trabajo fue financiado por FB 0002-

\section{LITERATURA CITADA}

Abarzúa, A. M. \& Moreno, P. I. (2008). Changing fire regimes in the temperate rainforest region of southern Chile over the last 16,000 yr. Quaternary Research, 69(1), 62-71.

Anderson, R. C. (1981). An evolutionary model summarizing the roles of fire, climate, and grazing animals in the origin and maintenance of grasslands: an end paper. In J. R. Estes, R. J. Tyrl \& J. N. Brunken (Eds) Grasses and grasslands: systematics and ecology (pp. 297-308). University of Oklahoma Press, Oklahoma.

Anderson, C. B., Griffith, C. R., Rosemond, A. D., Rozzi, R. \& Dollenz, O. (2006). The effects of invasive North American beavers on riparian plant communities in Cape Horn, Chile: do exotic beavers engineer differently in sub-Antarctic ecosystems?. Biological Conservation, 128, 467-474.

Anderson, C. B., Pastur, G., Lencinas, M.
V., Wallem, P. K., Moorman, M. C. \& Rosemond, A. D. (2009). Do introduced North American beavers Castor canadensis engineer differently in southern South America? An overview with implications for restoration. Mammal Review, 39, 33-52.

Armesto, J. J., Villagrán, C., Aravena, J. C., Pérez, C., Smith-Ramírez, C., Cortés, M. \& Hedin, L. O. (1995). Conifer forests of the Chilean coastal range. In R. S. Hill, N. Enright (Eds) Ecology of the Southern Conifers (pp. 156-170). Melbourne University Press, Melbourne.

Armesto, J. J., Bautista, S., del Val, E. Ferguson, B., García, X., Gaxiola, A., GodinezÁlvarez, H., Gann, G., López-Barrera, F., Manson, R., Núñez-Ávila, M., Ortiz-Arrona, C., Tognetti, P. \& Williams-Linera, G. (2007). Towards an ecological restoration network: Reversing land degradation in Latin America. Frontiers in Ecology and the Environment, 5(4), w1-w4.

Armesto, J. J., Smith-Ramírez, C., Carmona, M. R., Celis-Diez, J.L., Díaz, I. A., Gaxiola, A., Gutiérrez, A. G., Núñez-Avila, M. C., Pérez, C. A. \& Rozzi, R. (2009). Old-growth temperate rainforests of South America: Conservation, plant-animal interactions, and baseline biogeochemical processes. In C. Wirth ) Ed.) Old-growth forests (207, 367-390.) Springer-Verlag, Berlin.

Aronson, J., Clewell, A. F., Blignaut, J. N. \& Milton, S. J. (2006). Ecological Restoration: a new frontier for nature conservation and economics. Journal for Nature Conservation, 14, 135-139.

Bond, W. J. \& B. van Wilgen, W. (1996). Fire and plants. Population and community biology series, 14.

Bond, W. J. \& Keeley, J. E. (2005). Fire as a global 'herbivore': the ecology and evolution of flammable ecosystems. Trends in Ecology \& Evolution, 20(7), 387-394.

Bradshaw, A.D. (1983). The reconstruction of ecosystems. Journal of Applied Ecology, 20: $1-17$.

Chang, C. (1996). Ecosystem responses to fire and variations in fire regimes. In Sierra Nevada Ecosystem Project: Final Report to 
Congress (II, 1071-1099). Assessments and Scientific Basis for Management Options. Water Resources Center Report No. 37. Davis, CA. Centers for Water and Wildland Resources, University of California.

Clark, J. S., Stocks, B. J. \& Richard, P. J. H. (1996). Climate implications of biomass burning since the 19th century in eastern North America. Global Change Biology, 2, 433-442.

Comín, F. A., Menéndez, M., Pedrocchi, C., Moreno, S., Sorando, R., Cabezas, A., García, M.,... Ciancarelli, C. (2005). Wetland restoration: integrating scientifictechnical, economic, and social perspectives. Ecological Restoration, 23(3), 182-186.

Cowell, M. (2005). Ecological restoration and environmental ethics. Environmental Ethics, 15, 19-32.

Debano, L. F., Neary, D. G., Folliott, P. F. (1998). Fire's Effects on Ecosystems. John Wiley and Sons, New York.

Dobson, A. P., Bradshaw, A. D. \& Baker, A. J. M. (1997). Hopes for the future: restoration ecology and conservation biology. Science, 277, 515-522.

Donoso, C. (1993). Bosques templados de Chile y Argentina. Editorial Universitaria, Santiago, Chile.

Fernández, I., Morales, N., Olivares, L., Salvatierra, J., Gómez, M. \& Montenegro, G. (2010). Restauración ecológica para ecosistemas nativos afectados por incendios forestales. Facultad de Agronomía e Ingeniería Forestal, Pontificia Universidad Católica de Chile, Santiago, Chile.

Flannigan, M. D. \& Wotton, B. M. (2001). Climate, weather and area burned. In E. A. Johnson, K. Miyanishi (Eds.) Forest Fires: Behavior \& Ecological Effects (pp. 335-357). Academic Press, New York.

Fuentes, E. R. \& Espinoza, G. (1986). Resilience of shrublands in central Chile: a volcanismrelated hypothesis. Interciencia, 11, 164165.

Goldammer, J. G. (1991). Tropical wild-land fires and global changes: prehistoric evidence, present fire regimes, and future trends. In J. S. Levine (Ed.) Global biomass burning:
Atmospheric, climatic, and biospheric implications. (pp. 83-91). Massachusetts Institute of Technology Press, Cambridge.

González, M. E. \& Veblen, T. T. (2006). Climatic influences on fire in Araucaria araucanaNothofagus forests in the Andean cordillera of south-central Chile. Ecoscience, 13(3), 342-350.

Henn J. J., Anderson, C. B., Kreps, G., Lencinas, M. V., Soler, R. \& Martínez Pastur, G. (2014). Determining abiotic and biotic factors that limit transplanted Nothofagus pumilio seedling success in abandoned beaver meadows in Tierra del Fuego. Ecological Restoration, 32 (4), 369-378.

Heusser, C. J. (1987). Fire history of FuegoPatagonia. Quaternary of South America and Antarctic Peninsula, 5, 93-109.

Heusser, C. J. (1994). Paleoindians and fire during the Late Quaternary in southern South America. Revista Chilena de Historia Natural, 67, 435-443.

Heusser, C. J. (1995). Three Late Quaternary pollen diagrams from Southern Patagonia and their palaeoecological implications. Palaeogeography, Palaeoclimatology, Palaeoecology, 118, 1-24.

Higgs, E. (2005). The two-culture problem: ecological restoration and the integration of knowledge. Ecological Restoration, 13, 159-164.

Huber, U. M., Markgraf, V. \& Schäbitz, F. (2004). Geographical and temporal trends in Late Quaternary fire histories of FuegoPatagonia, South America. Quaternary Science Reviews, 23(9), 1079-1097.

Kitzberger, T., Veblen, T. T. \& Villalba, R. (1997). Climatic influences on fire regimes along a rainforest-to-xeric woodland gradient in northern Patagonia, Argentina. Journal of Biogeography, 24, 35-47.

Lara, A., Soto, D., Armesto, J. J., Donoso, P., Wernli, C., Nahuelhual, L. \& Squeo, F. (2003). Componentes científicos clave para una política nacional sobre usos, servicios y conservación de los bosques nativos chilenos. Universidad Austral de Chile, Iniciativa Científica Milenio de MIDEPLAN, Valdivia. 
Leopold, B. D., Maehr, D. S., Noss, R. F. \& Larkin, J. L. (2002). Large mammal restoration: ecological and sociological challenges in the 21st century. Restoration Ecology, 10, 732-733.

Lloret, F. \& Vilà, M. (2003). Diversity patterns of plant functional types in relation to fire regime and previous land use in Mediterranean woodlands. Journal of Vegetation Science, 14(3), 387-398.

Long, C. J., Whitlock, C., Bartlein, P. J. \& Millspaugh, S. H. (1998). A 9,000-year fire history from the Oregon Coast Range, based on a high-resolution charcoal study. Canadian Journal of Forest Research, 28(5), 774-787.

Markgraf, V. \& Anderson, L. (1994). Fire history of Patagonia: climate versus human cause. Revista do Instituto Geológico, 15(12), 35-47.

McIntosh, R. P. 1980. The background and some current problems of theoretical ecology. Synthèse, 43(2), 195-255.

Mermoz, M., Kitzberger, T. \& Veblen, T. T. (2005). Landscape influences on occurrence and spread of wildfires in Patagonian forests and shrublands. Ecology, 86(10), 2705-2715.

Montenegro, G., Ginocchio, R., Segura, A., Keely, J. E. \& Gómez, M. (2004). Regímenes de incendios y respuestas de la vegetación en dos regiones de clima mediterráneo. Revista Chilena de Historia Natural, 77(3), 455464.

Moreno, P. I. (2004). Millennial-scale climate variability in northwest Patagonia during the last 15,000 yr. Journal of Quaternary Science, 19, 35-47.

Moreno, P. I., Kaplan, M. R., Francois, J. P., Villa-Martínez, R., Moy, C. M., Stern, C. R. \& Kubik, P. W. (2009). Renewed glacial activity during the Antarctic cold reversal and persistence of cold conditions until $11.5 \mathrm{ka}$ in southwestern Patagonia. Geology, 37(4), 375-378.

Morrison, E. B. \& Linderll, C. A. (2010). Active or passive restoration? Assessing restoration alternatives with avian foraging behavior. Restoration Ecology, 19, 170-177.

Mutch, R. W. (1970). Wildland fires and ecosystems-a hypothesis. Ecology, 10461051.

Palmer, M. A., Ambrose, R. F. \& Poff, N. L. (1997). Ecological theory and community restoration ecology. Restoration Ecology, 5, 291-300.

Power, M. J., Marlon, J., Ortiz, N., Bartlein, P. J., Harrison, S. P., Mayle, F. E., Ballouche, A.,... Zhang, J. H. (2008). Changes in fire regimes since the Last Glacial Maximum: an assessment based on a global synthesis and analysis of charcoal data. Climate Dynamics, 30(7-8), 887-907.

Ruwanza, W., Gaertner, M., Esler, K. J. \& Richardson, D. M. (2013). The effectiveness of active and passive restoration on recovery of indigenous vegetation in riparian zones in the Western Cape South Africa: a preliminary assessment. South African Journal of Botany, 88, 132-141.

Sanhueza, P. I. (2001). Fire situation in Chile. In J. G. Goldammer \& R. W. Mutch (Eds.) Global forest fire assessment. (pp.1990-2000). Forest Resources Assessment, FAO, Rome. Shlisky, A., Waugh, J., Gonzalez, P., Gonzalez, M., Manta, M., Santoso, H., Alvarado, E.,... Zollne, D. (2007). Fire, ecosystems and people: threats and strategies for global biodiversity conservation. The Nature Conservancy, Arlington, DC.

Tortorelli, L. A. (1947). Los incendios de bosques en la Argentina. Ministerio de Agricultura de la Nación. Argentina: Dirección Forestal.

van Andel, J. \& Aronson, L. (2006). Restoration Ecology. Blackwell Publishing, UK.

Veblen, T. T., Burns, B. R., Kitzberger, T., Lara, A. \& Villalba, R. (1995). The ecology of the conifers of southern South America. In N. J. Enright, R. S. Hill (Eds.) Ecology of the Southern conifers. (pp. 120-155). Melbourne University Press, Australia.

Veblen, T. T., Kitzberger, T., Villalba, R. \& Donnegan, J. (1999). Fire history in northern Patagonia: the roles of humans and climatic variation. Ecological Monographs, 69, 47-67.

Veblen, T. T., Holz, A., Paritsis, J., Raffaele, E., Kitzberger, T. \& Blackhall, M. (2011). Adapting to global environmental change 
in Patagonia: What role for disturbance ecology? Austral Ecology, 36(8), 891903.

Villa-Martínez, R. \& Moreno, P. I. (2007). Pollen evidence for variations in the southern margin of the westerly winds in SW Patagonia over the last 12,600 years. Quaternary Research, 68, 400-409.

Walker, L. R. \& Moral, R. (2003). Primary succession and ecosystem rehabilitation. Cambridge University Press, Cambridge, UK.

Wallem, P. K., Jones, C. G., Marquet, P. A. \& Jaksic, F. M. (2007). Identificación de los mecanismos subyacentes a la invasión de Castor canadensis (Rodentia) en el archipiélago de Tierra del Fuego, Chile. Revista Chilena de Historia Natural, 80, 309-325.

Whelan, R. J. (1995). The Ecology of Fire. Cambridge University Press, Cambridge, UK.

White, P. S. \& Walker, J. L. (1997). Approximating
Nature's Variation: Selecting and Using Reference Information in Restoration Ecology. Restoration Ecology, 5(4), 338-349.

Whisenant, S. G. (1999). Repairing damaged wildlands: a process oriented, landscapescale approach. Cambridge University Press, Cambridge, UK.

Whitlock, C., Bianchi, M. M., Bartlein, P. J., Markgraf, V., Marlon, J., Walsh, M. \& McCoy, N. (2006). Postglacial vegetation, climate, and fire history along the east side of the Andes (lat 41-42.5 S), Argentina. Quaternary Research, 66(2), 187-201.

Whitlock, C., P. I. Moreno \& Bartlein, P. (2007). Climatic controls of Holocene fire patterns in southern South America. Quaternary Research, 68, 28-36.

Young, T. P., Chase, J.M. \& Huddleston, R. T. (2001). Community succession and assembly: comparing, contrasting and combining paradigms in the context of ecological restoration. Ecological Restoration, 19, 5-18. 